\title{
Inclusive b-jet production in heavy ion collisions at the LHC
}

\author{
Jinrui Huang** Zhong-Bo Kang $\oplus^{*}$ and Ivan Vitev \\ Theoretical Division, Los Alamos National Laboratory, Los Alamos, NM 87545, USA
}

(Dated: October 9, 2013)

\begin{abstract}
Theoretical and experimental advances in understanding light jet production and modification in $\mathrm{Pb}+\mathrm{Pb}$ reactions at $\sqrt{s_{N N}}=2.76 \mathrm{TeV}$ have been a highlight of the LHC heavy ion program. At the same time, the detailed mechanisms of heavy quark propagation and energy loss in dense QCD matter are not yet fully understood. With this motivation, we present theoretical predictions for the nuclear-induced attenuation of the differential cross section for inclusive b-jet production in heavy ion collisions at the LHC for comparison to upcoming data. We find that for transverse momenta $p_{T} \gtrsim 75 \mathrm{GeV}$ both hadronization and mass effects are negligible and this attenuation is comparable to the one observed for light jets. We discuss how the detailed b-jet quenching patterns can be used to gain new insight into the in-medium heavy flavor dynamics.

PACS numbers: 12.38.Bx, 12.38.Mh, 13.87.-a
\end{abstract}

\section{INTRODUCTION}

Collimated jets of hadrons are a dominant feature of high energy particle interactions. They have long been regarded as the main tools for testing the fundamental properties of Quantum Chromodynamics (QCD), for obtaining information about the structure of the proton, and for searching for new physics beyond the Standard Model [1]. The current highest energy hadron collider, the Large Hadron Collider (LHC) at CERN, guarantees an abundant yield of large transverse momentum jets [24.

High energy jets also provide unprecedented opportunities to study the properties of dense QCD matter, cold nuclear matter (CNM) and the quark-gluon plasma (QGP) that is created in the ultra-relativistic collisions of heavy nuclei. In such reactions, large transverse momentum partons traverse a medium composed of colored quasi-particles and lose energy via induced gluon radiation and elastic scattering. This "jet quenching" phenomenon modifies the observables associated with jets relative to the vacuum case [5]. The differences can be used to gain insight into the mechanisms of parton interactions in dense QCD matter and to provide deeper understanding of the in-medium parton shower formation. For this reason, inclusive and tagged jet production in $\mathrm{p}+\mathrm{p}$ collisions and the corresponding modification in $\mathrm{Pb}+\mathrm{Pb}$ reactions have been intensively investigated by both the ATLAS and the CMS collaborations at the LHC [6, 7]. ALICE collaboration results are also expected in the near future. Theoretical predictions and description of the data based on models of "jet quenching" are in good agreement with the experimental results 8 12], providing valuable information on the light flavor parton dynamics in the QGP.
Although these successful descriptions are rather encouraging, there are still remaining open questions. One well-known difficulty is related to the fact that the heavy quark (charm and bottom) parton-level energy loss has not been sufficient in the past to explain the observed suppression in the heavy mesons (or $e, \mu$ coming from their semi-leptonic decays) at both the RHIC and the LHC [13, 14]. There are extensive theoretical attempts aiming to explain the observed suppression [1519]. Meanwhile, additional experimental observables are also being proposed to further test the heavy flavor energy loss mechanisms [20].

In this paper, we take a different approach and present a study of inclusive heavy flavor jet production in heavy ion collisions. More specifically, we report first results and predictions for the inclusive b-jet cross sections in $\sqrt{s_{N N}}=2.76 \mathrm{TeV} \mathrm{Pb}+\mathrm{Pb}$ collisions at the LHC. We combine consistently the simulations in nucleon-nucleon reactions, which are validated through comparison to the $\sqrt{s}=7 \mathrm{TeV} \mathrm{p}+\mathrm{p}$ data at the LHC, with CNM effects and parton energy loss effect in the QGP. We study the relation between the attenuation of inclusive b-jet production and the physics of heavy quark production and propagation in dense QCD matter. Predictions on the nuclear modification factor $R_{A A}$ as a function of the jet transverse momentum $p_{T}$ and the jet radius parameter $R$ are given.

The rest of our paper is organized as follows: in Sec. II we present the evaluation of the inclusive b-jet cross section in $\mathrm{p}+\mathrm{p}$ collisions using Pythia simulations. In Sec. III we describe the simulation of the mediuminduced parton shower and the related heavy quark mass effects. Our phenomenological results for the b-jet production in the $\mathrm{Pb}+\mathrm{Pb}$ collisions at the $\mathrm{LHC}$ are given in Sec. IV. We conclude our paper in Sec. V.

\footnotetext{
* jinruih@lanl.gov

† zkang@lanl.gov

$\ddagger$ ivitev@lanl.gov
} 


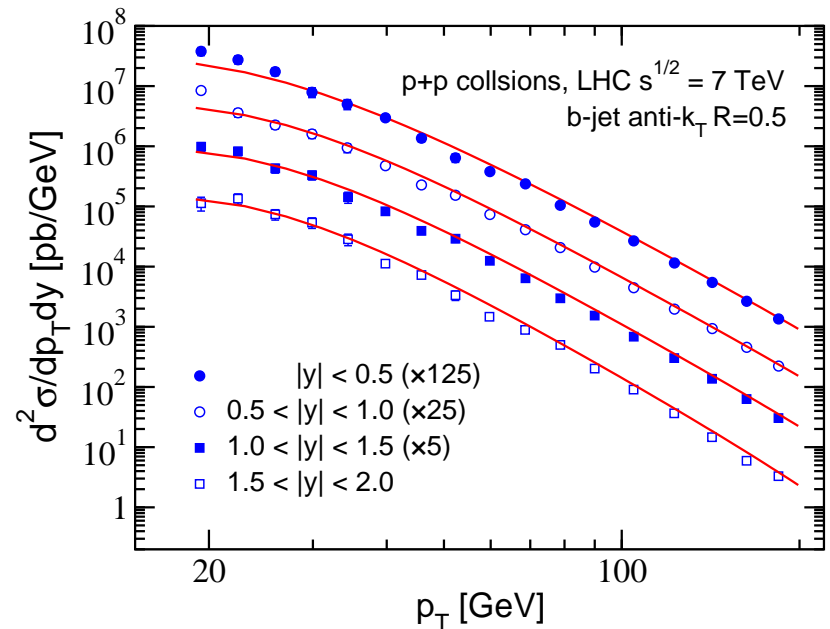

FIG. 1. Differential cross sections versus $p_{T}$ are shown for inclusive b-jets in four different rapidity $y$ regions at the LHC, $\sqrt{s}=7 \mathrm{TeV} \mathrm{p}+\mathrm{p}$ collisions. Red lines are from the Pythia 8 simulations, using the anti- $k_{T}$ jet algorithm with $R=0.5$. Circles, open-circles, squares and open-squares correspond to the CMS experimental results [25].

\section{INCLUSIVE B-JET PRODUCTION IN P+P COLLISIONS}

In this section, we present the evaluation of the inclusive b-jet cross section in $\mathrm{p}+\mathrm{p}$ collisions using Pythia 8 [21]. Specifically, the Pythia 8 event generator with the CTEQ6L1 parton distribution functions [22] is employed to simulate the inclusive jet events in the $\mathrm{p}+\mathrm{p}$ collisions for center-of-mass energies $\sqrt{s}=7 \mathrm{TeV}$ and $\sqrt{s}=2.76 \mathrm{TeV}$. This generator utilizes leadingorder perturbative QCD matrix element for $2 \rightarrow 2$ processes, combined with a leading-logarithmic $p_{T}$-ordered parton shower, and the Lund string model for hadronization. Furthermore, the SlowJet program is used for the jet clustering with an anti- $k_{T}$ algorithm [23], which has been checked to yield the same results as FastJet [24]. The b-jets are defined to be jets that contain at least one b-quark (or $\bar{b}$-quark) inside the jet cone. Thus, to further identify the b-jets from the inclusive jets found by the SlowJet, a b-quark (or $\bar{b}$-quark) is assigned to a jet if the radial separation from the reconstructed jet axis is $\Delta R<R . \quad R$ is the jet radius parameter and $\Delta R=\sqrt{(\Delta \phi)^{2}+(\Delta y)^{2}}$, where $\phi$ and $y$ are the azimuthal angle and the rapidity, respectively.

In Fig. 1, we evaluate the inclusive b-jet differential cross section $d^{2} \sigma / d y d p_{T}$ with $R=0.5$ in $\mathrm{p}+\mathrm{p}$ collisions at $\sqrt{s}=7 \mathrm{TeV}$ as a function of jet transverse momentum $p_{T}$ at different rapidity regions using Pythia 8 (red lines). We compare the simulations to recent CMS experimental data (circle, open-circle, square and open-square data points) [25]. We find that for all four rapidity regions the Pythia simulations agree well with the experimental data. Note, that we have included hadronization effects in this comparison. Let us elaborate further on such ef- fects. Since all interactions in dense QCD matter that we consider in this paper happen at the partonic level, it is important to assess how hadronization affects the b-jet cross section. In Pythia simulations one can easily turn off hadronizaiton effects, but still keep the parton shower and obtain the b-jet cross section from the clustering of the final-state partons. We find that, for all jet radii relevant to our study $(0.2 \leq R \leq 0.7)$, noticeable effects of hadronization exist only at relatively low transverse momentum $\left(p_{T} \lesssim 30 \mathrm{GeV}\right)$ and small jet radius parameter $R=0.2,0.3$. This is consistent with the basic premises of jet physics, namely, that it accurately reflects the parton level QCD dynamics. Since we are mainly interested in the high $p_{T}$ region, the hadronization effects are negligible in our study of nuclear modification of the b-jet production.

In the following sections we will present the nuclear modification of b-jet production in $\mathrm{Pb}+\mathrm{Pb}$ collisions at $\sqrt{s_{N N}}=2.76 \mathrm{TeV}$. In order to study energy loss effects and the medium-induced parton shower, we need detailed information on the flavor content of the b-jets produced in the $\mathrm{p}+\mathrm{p}$ collisions at $\sqrt{s}=2.76 \mathrm{TeV}$. Heavy quark production in hadronic collisions can be generated through various mechanisms [26] that (in the fixed flavor scheme [27]) can be categorized into three classes: "heavy quark pair creation", "gluon splitting", and all other "light quark pair creation" processes. In Pythia simulations, these mechanisms can be easily separated and studied through the $2 \rightarrow 2$ hard partonic scattering, showering, and hadronization. In the so-called "heavy quark pair creation" process, two heavy quarks are produced in the hard subprocess. At leading order this is described by $g g \rightarrow b \bar{b}$ and $q \bar{q} \rightarrow b \bar{b}$. In this case, one of the b-quark (b-quark) initiates the b-jet. In heavy ion collisions, the medium modification of such b-jet behaves more like a single b-quark transversing the QGP, and thus has a direct connection to the heavy quark energy loss [29]. On the other hand, the "gluon splitting" mechanism can also generate b-jets, in which heavy quarks do not participate in the hard subprocesses, but are produced through the $g \rightarrow b \bar{b}$ channel in the parton shower. In Pythia simulation, we collect $g g \rightarrow g g, q g \rightarrow q g$, and $q \bar{q} \rightarrow g g$ in this category. The time for such gluon splitting, $\left[p^{+}, 0, \mathbf{0}_{\perp}\right]_{g} \rightarrow\left[z p^{+},\left(\mathbf{k}^{2}+m_{b}^{2}\right) / 2 z p^{+}, \mathbf{k}\right]_{b}+[(1-$ $\left.z) p^{+},\left(\mathbf{k}^{2}+m_{b}^{2}\right) / 2(1-z) p^{+},-\mathbf{k}\right]_{\bar{b}}$, can be estimated from the inverse virtuality for this process to occur [15]:

$$
\tau_{\text {split }}=\frac{1}{1+\beta_{b \bar{b}}} \frac{2 z(1-z) p^{+}}{\mathbf{k}^{2}+m_{b}^{2}}
$$

In Eq. (11) $p^{+}$is the large lightcone momentum of the parton, the transverse momentum $|\mathbf{k}| \sim \Lambda_{\mathrm{QCD}}$ is perpendicular to the direction of the jet, and $\beta_{b \bar{b}}$ is the velocity of the heavy $b \bar{b}$ pair (we take $m_{b}=4.5 \mathrm{GeV}$ ). Even for jets of transverse momentum of $200 \mathrm{GeV}$ at midrapidity $\left(p^{+}=400 \mathrm{GeV}\right)$ the splitting time is $\tau_{\text {split }}<1 \mathrm{fm}$, much smaller than the typical size of the medium $\sim R_{P b}$. In heavy ion collisions, the medium modification of these bjets would resemble that of a massive gluon (color octet 


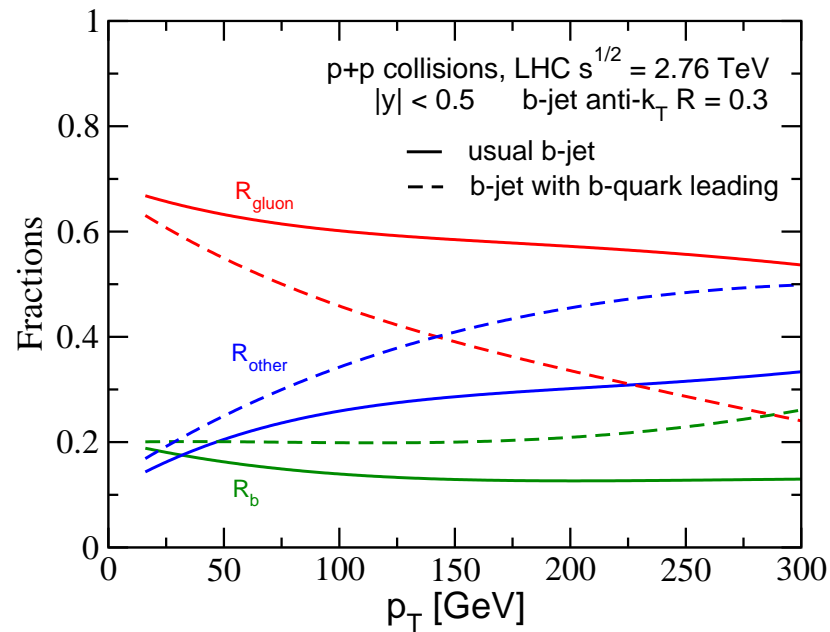

FIG. 2. The relative contribution of different subprocesses to the inclusive b-jet production cross section in $\mathrm{p}+\mathrm{p}$ collisions at the LHC with $\sqrt{s}=2.76 \mathrm{TeV}$. Here, b-jet production is simulated using Pythia 8 with an anti- $k_{T}$ jet algorithm and $R=0.3$, and we have integrated over the central rapidity region $|y|<0.5$. The solid curves are for the commonly defined b-jets, while the dashed curves further require that the contained b-quark (or $\bar{b}$-quark) is the leading particle in the jet.

state) transversing the QGP [30]. Finally, all other partonic channels can also generate b-jets through the parton shower. These include $g g \rightarrow q \bar{q}, q q \rightarrow q q$, and $q \bar{q} \rightarrow q \bar{q}$ in the Pythia simulations. Following the same logic, they will behave like a massive quark (color triplet state) transversing the QGP in the case of heavy ion collisions. We denote the fractional contribution to the b-jet cross section from the "heavy quark pair creation" as $R_{b}$, the "gluon splitting" as $R_{g}$, and the remaining contributions as $R_{\text {other }}$.

In Fig. 2, we show these relative contributions as a function of the usual b-jet transverse momentum $p_{T}$ for $\sqrt{s}=2.76 \mathrm{TeV} \mathrm{p}+\mathrm{p}$ collisions with $R=0.3$ and jet rapidity $|y|<0.5$ (the solid curves). We find that the contribution from "gluon splitting" is dominant in all of the studied $p_{T}$ range, while the "heavy quark pair creation" contribution is only around $10 \%-20 \%$, depending on the $p_{T}$ range. Our relative fractions $R_{b}, R_{g}$, and $R_{\text {other }}$ are roughly consistent with previous simulations based on Herwig [31]. Naively, one would like the nuclear modification of b-jets to be related to the dense QCD matter effects (energy loss) of a leading b-quark. This implies that one has to increase the contribution from "heavy quark pair creation", as we have explained above. We find that if we require that the contained b-quark (or $\bar{b}$ quark) is also the leading particle inside the jet cone, the fraction $R_{b}$ can increase quite a bit at high $p_{T}$ and reach $\sim 30 \%$ at $p_{T} \sim 300 \mathrm{GeV}$, which is still subdominant. To further increase the contribution from the "heavy quark pair creation", one realizes the following: for b-jets that originate from heavy flavor creation, only one b-quark (or $\overline{\mathrm{b}}$-quark) is expected to be inside the same jet cone. On the other hand, for b-jets that originate from gluon splitting, the b-b quark pair is likely collimated and, hence, clustered in the same jet. To reduce the relative contribution from "gluon splitting", one can study b-jets which contain only a single b-quark inside the jet cone, as has already been done by the CDF collaboration at the Tevatron [32]. Nuclear modification of such "single b-quark jet" might reflect better the physics of heavy quark energy loss in the QGP.

\section{MODIFICATION OF B-JET PRODUCTION RATES IN DENSE QCD MATTER}

The baseline for the evaluation of the b-jet cross sections in heavy ion collisions are the corresponding cross sections in the more elementary nucleon-nucleon reactions. In Ref. [33] it was shown that in QCD the finalstate process-dependent medium-induced radiative corrections factorize in the production cross sections for the example of a single jet,

$$
d \sigma(\epsilon)_{\text {quench. }}^{\mathrm{jet}}=d \sigma(\epsilon)_{\mathrm{CNM}}^{\mathrm{jet}} \otimes P(\epsilon)|J(\epsilon)| .
$$

In Eq. (2) $d \sigma(\epsilon)_{\mathrm{CNM}}^{\mathrm{jet}}$ contains only cold nuclear matter effects and the differential phase space factors are omitted for brevity. Furthermore, the functions above depend on the kinematics, such as the jet $p_{T}$ and $y$, which we don't write explicitly. $P(\epsilon)$ is the probability that the hard parent parton will redistribute a fraction of its energy $\epsilon$ into a medium-induced parton shower, and $\otimes$ denotes an integral convolution. $|J(\epsilon)| \equiv\left|J\left(\epsilon ; R, \omega^{\text {coll }}\right)\right|$ is a phase space Jacobian that further takes into account what fraction of the medium-induced parton shower energy is retained inside the jet cone of radius $R$, as opposed to "lost" outside. The reason that formation of an in-medium parton shower ultimately leads to a suppression of the jet cross section is two-fold. First, this shower has a wider angular distribution relative to the vacuum shower. This is true both in the soft gluon approximation that we use in this paper and for the full $\mathcal{O}\left(\alpha_{s}\right)$ medium-induced splittings [34]. We recently checked on the example of $q \rightarrow q g g$ that the broad angular distribution feature of in-medium branchings holds to $\mathcal{O}\left(\alpha_{s}^{2}\right)$ [35]. Second, the partons of the in-medium parton shower can further transfer their energy to the stronglyinteracting plasma through collisional interactions at a rate $\frac{d E^{\text {coll }}}{d \Delta z} \propto \frac{\alpha_{s}^{\text {med }} C_{(s)} m_{D}^{2}}{2} \ln \frac{\sqrt{E T}}{m_{D}}$ to leading logarithmic accuracy [36]. Here, $m_{D}=g^{\text {med }} T$ is the Debye screening mass for a gluon-dominated plasma of temperature $T$ and $C_{(s)}$ is the quadratic Casimir for the color representation of the corresponding state: $C_{(3)}=C_{F}=4 / 3$, $C_{(8)}=C_{A}=3$. This energy is also transported away from the direction of the jet through the excitations of the medium.

The starting point for the evaluation of the QGP effects in Eq. (2) is the medium-induced gluon distribution, 
which we calculate to first order in opacity [37]:

$$
\begin{aligned}
k^{+} \frac{d^{3} N^{g}}{d k^{+} d^{2} \mathbf{k}}= & \frac{C_{(s)} \alpha_{s}}{\pi^{2}} \int \frac{d \Delta z}{\lambda_{(8)}} \int d^{2} \mathbf{q} \frac{1}{\sigma_{e l}} \frac{d^{2} \sigma_{e l}}{d^{2} \mathbf{q}} \frac{2 \mathbf{k} \cdot \mathbf{q}}{\mathbf{k}^{2}(\mathbf{k}-\mathbf{q})^{2}} \\
& \times\left(1-\cos \left(\frac{(\mathbf{k}-\mathbf{q})^{2}}{k^{+}} \Delta z\right)\right) .
\end{aligned}
$$

In Eq. (3), $k^{+}$is the large lightcone momentum of the emitted gluon in the direction of the hard parent parton, $\mathbf{k}$ is its momentum transverse to the jet axis, and $\mathbf{q}$ is the transverse momentum exchange between the propagating system and the QCD medium. $\frac{1}{\sigma_{e l}} \frac{d^{2} \sigma_{e l}}{d^{2} \mathbf{q}}$ is the normalized differential distribution of the transverse momentum transfers. Note that, as written, Eq. (3) relates to massless partons. Thermal effects in the subasymptotic temperature regime are included with an effective mass $\mathbf{k}^{2} \rightarrow \mathbf{k}^{2}+m_{D}^{2}$. The details of evaluating the double differential distribution $k^{+} \frac{d^{3} N^{g}}{d k^{+} d^{2} \mathbf{k}}$ are given elsewhere [5, 37]. We point out that, in order to calculate the bremsstrahlung of a collimated multi-parton system that is not resolved by the momentum exchanges in the medium, we have generalized Eq. (3) to relate the color state $(s)$ to the representation of the parent parton. For example, for $g \rightarrow b \bar{b},(s)=(8)$ and $q \rightarrow q g \rightarrow q b \bar{b}$, $(s)=(3)$. The mass $M=m_{b}, 2 m_{b}$ is implemented as follows: $\mathbf{k}^{2} \rightarrow \mathbf{k}^{2}+x^{2} M^{2}$, where $x=k^{+} / p^{+}$is the lightcone momentum fraction of the gluon $\left(k^{+} \approx 2 \omega\right.$, $\left.p^{+}=2 E\right)$. An example of such application for a different observable will be the quenching of the color-octet contribution to quarkonium production at high transverse momentum [30].

With the QGP-induced distribution of gluons $\frac{d^{3} N^{g}}{d k^{+} d^{2} \mathbf{k}}$ and the related $\frac{d^{2} N^{g}}{d \omega d r}$ ( $\omega$ is the energy and $r$ is the angle) of gluons at hand, we can evaluate the fraction $f$ of the in-medium parton shower energy that is contained in the jet cone of radius $R$ :

$$
f\left(R, \omega^{\mathrm{coll}}\right)_{(s)}=\frac{\int_{0}^{R} d r \int_{\omega^{\mathrm{coll}}}^{E} d \omega \frac{\omega d^{2} N_{(s)}^{g}}{d \omega d r}}{\int_{0}^{R^{\infty}} d r \int_{0}^{E} d \omega \frac{\omega d^{2} N_{(s)}^{g}}{d \omega d r}} .
$$

In Eq. (41) $f(R, 0)_{(s)}$ takes into account mediuminduced parton splitting effects. On the other hand $f\left(R^{\infty}, \omega^{\text {coll }}\right)_{(s)}=\Delta E^{\text {coll }} / E$ is the energy dissipated by the medium-induced parton shower into the QGP due to collisional processes. $\Delta E^{\text {coll }}$ is evaluated as in Ref. 36] and helps solve for $\omega^{\text {coll }}$. Then, for any $R$, Eq. (4) allows to treat the radiative and collisional energy loss effects on the same footing.

Writing down explicitly the phase space Jacobian $|J(\epsilon)|_{(s)}=1 /\left(1-\left[1-f\left(R, \omega^{\text {coll }}\right)_{(s)}\right] \epsilon\right)$ and applying Eq. (2) to the case of b-jets we obtain the cross section per elementary nucleon-nucleon collision:

$$
\begin{aligned}
\frac{1}{\left\langle N_{\text {bin }}\right\rangle} \frac{d^{2} \sigma_{A A}^{\mathrm{b}-\mathrm{jet}}(R)}{d y d p_{T}} & =\sum_{(s)} \int_{0}^{1} d \epsilon \frac{P_{(s)}(\epsilon)}{\left(1-\left[1-f\left(R, \omega^{\mathrm{coll}}\right)_{(s)}\right] \epsilon\right)} \\
& \times \frac{d^{2} \sigma_{(s)}^{\mathrm{CNM}, \mathrm{LO}+\mathrm{PS}}\left(|J(\epsilon)|_{(s)} p_{T}\right)}{d y d p_{T}}
\end{aligned}
$$

Here, the sum runs over the set of final states (s) described in Sec. II. Their relative contribution as a function of $p_{T}$ was presented in Fig. 2. $d^{2} \sigma^{\mathrm{CNM}, \mathrm{LO}+\mathrm{PS}} / d y d p_{T}$ includes cold nuclear matter effects. Note that, just like in the QGP, we consider physics related to the coherent, elastic and inelastic parton scattering in large nuclei [38]. Of these effects, at the transverse momenta and virtualities for the hard parton scattering subprocess that we consider, only initial-state energy loss may play a role 37. As we will see in the next section, the influence of CNM effects on b-jet production is relatively small.

In reactions with heavy nuclei one has to take into account the geometry of the collision. The hard parent parton production points are distributed in the transverse plane according to the binary collision density $\sim$ $d^{2} N_{\text {bin }} / d^{2} \mathbf{x}$. In contrast, the gluon-dominated medium is assumed to follow the number of participants density $\sim d^{2} N_{\text {part }} / d^{2} \mathbf{x}$ and undergoes longitudinal Bjorken expansion. Specifically, we use an optical Glauber model with an inelastic scattering cross section $\sigma_{i n}=64 \mathrm{mb}$.

\section{PHENOMENOLOGICAL RESULTS}

We now turn to the phenomenological results for b-jet production in $\mathrm{Pb}+\mathrm{Pb}$ collisions at the LHC. Our simulations are performed at a center-of-mass energy per nucleon-nucleon pair $\sqrt{s_{N N}}=2.76 \mathrm{TeV}$ for direct comparison to upcoming experimental measurements at the LHC. Unless otherwise specified, we focus on the most central collisions with average number of participants $N_{\text {part }}=360$. As discussed in the previous section, the redistribution of the energy of a hard scattered parton in the final state between a vacuum and a medium-induced parton shower will lead to a modification of the observed b-jet cross section. It can be quantified through the nuclear modification ratio:

$$
R_{A A}^{\mathrm{b}-\text { jet }}\left(p_{T} ; R\right)=\frac{\frac{d^{2} \sigma^{A A}\left(p_{T} ; R\right)}{d y d p_{T}}}{\left\langle N_{\mathrm{bin}}\right\rangle \frac{d^{2} \sigma^{p p}\left(p_{T} ; R\right)}{d y d p_{T}}} .
$$

Here, $d^{2} \sigma^{p p}\left(p_{T} ; R\right)$ and $d^{2} \sigma^{A A}\left(p_{T} ; R\right)$ are the differential cross sections in $\mathrm{p}+\mathrm{p}$ and $\mathrm{A}+\mathrm{A}$ reactions respectively and $\left\langle N_{\text {bin }}\right\rangle$ is the average number of nucleon-nucleon scatterings for a given centrality class.

Since a number of CNM and QGP effects can contribute to the $R_{A A}$ of b-jets, we will study these effects separately to demonstrate how they can be identified and constrained from the experimental measurements. Let us first concentrate on the redistribution of the hard parton energy between the vacuum and the medium-induced shower due to soft gluon emission. The physics of jet cross section suppression lies in the broader angular distribution of the medium-induced parton shower [5]. Consequently, one expects that the quenching effect will be the largest for the smallest jet radius $R$ and the effect will disappear $\left(R_{A A} \rightarrow 1\right)$ for very large radii. This 


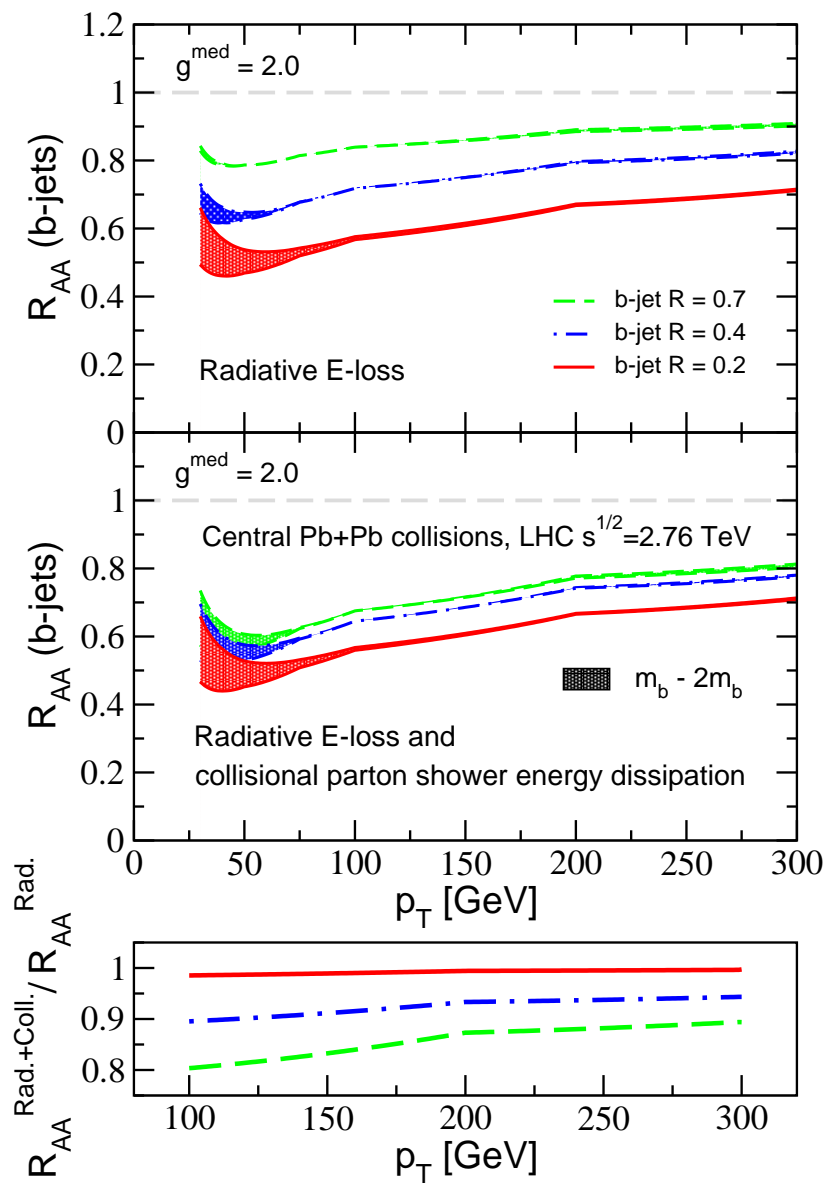

FIG. 3. The predicted jet radius $R$ dependence of the nuclear suppression for b-jet production in central $\mathrm{Pb}+\mathrm{Pb}$ collisions at the $\mathrm{LHC}$ at $\sqrt{s_{N N}}=2.76 \mathrm{TeV}$ is shown verses the jet transverse momentum. We have chosen radii $R=0.2,0.4,0.7$ and a coupling between the jet and the medium $g^{\text {med }}=2$. The upper panel only shows the effect of radiative energy loss and the lower panel includes the collisional dissipation of the parton shower energy in the QGP. Bands correspond to a range of masses of the propagating system between $m_{b}$ and $2 m_{b}$. The bottom insert shows the ratio of $R_{A A}$ s for radiative + collisional energy loss and radiative energy loss only.

is shown in the upper panel of Fig. 3 for coupling between the jet and the medium $g^{\text {med }}=2$ (corresponding to $\alpha_{s}^{\text {med }}=0.32$ ). We concentrate on the region of $p_{T}>30 \mathrm{GeV}$ where hadronization corrections for bjets are minimal even for small radii. The jet radius effect of jet quenching is clearly seen by comparing the magnitude of the jet suppression for three different radii, $R=0.2$ (red solid line), $R=0.4$ (blue dot-dashed line), and $R=0.7$ (light green dashed line). The bands correspond to a range of masses for the collimated propagating parent parton system $\left(m_{b}, 2 m_{b}\right)$. The bottom insert in Fig. 3 shows the ration $R_{A A}^{\text {Rad. }+ \text { Coll. }} / R_{A A}^{\text {Rad. }}$ to clarify the significance of the collisional energy loss for different b-jet radii.

Note, that above $p_{T}=75 \mathrm{GeV}$ the mass effect disappears even for $2 m_{b}=9 \mathrm{GeV}$. This is a direct consequence

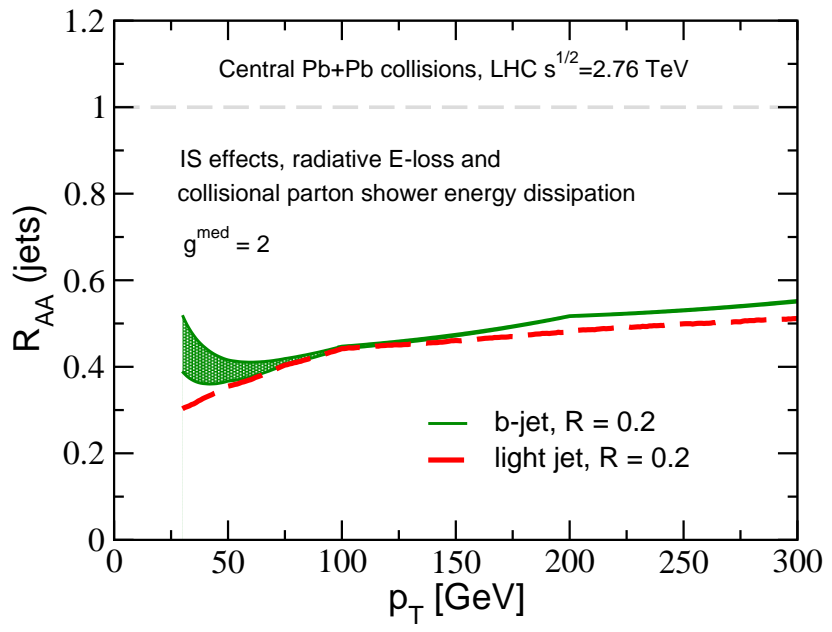

FIG. 4. The $p_{T}$-dependent suppressions of both b-jet and light jet cross sections in central $\sqrt{s_{N N}}=2.76 \mathrm{TeV} \mathrm{Pb}+\mathrm{Pb}$ collisions at the LHC are shown for radius $R=0.2$ and the coupling between the jet and the medium $g^{\text {med }}=2$. The band for b-jet is the same as in Fig. 3 .

of the fully coherent energy loss regime. For incoherent bremsstrahlung, just like in QED, the mass effect never vanishes [37]. Thus, observation of b-jet quenching comparable to that of light jets at transverse momenta $p_{T}>75 \mathrm{GeV}$ will constitute direct experimental evidence for the dominance of Landau-Pomeranchuk-Migdal type destructive interference effects in the medium-induced parton shower formation. Below $p_{T}$ of $75 \mathrm{GeV}$, there is a distinct trend toward reduction of the jet suppression. The reason for this reduction in quenching is two-fold. On one hand, below $75 \mathrm{GeV}$ the b-quark mass starts to play a role. On the other hand, the b-jet spectra stiffen considerably. Finally, there is a modest $p_{T}$ dependence of $R_{A A}$ up to transverse momenta of $300 \mathrm{GeV}$. These features are clearly shown in Fig. 4, where a comparison for the nuclear suppression between b-jet and light jet is presented. The $R_{A A}$ for light-jet production is directly taken from previous work [9]. The tiny difference at high $p_{T}$ is smaller than the uncertainty in the treatment of cold nuclear matter effects and collisional energy loss between these two cases.

In the lower panel of Fig. 3 we present a similar calculation but include the collisional dissipation of the mediuminduced parton shower energy in the QGP. This dissipation is evaluated as in [36], including the interference between the parent parton and the radiated gluon, and implemented as thermalization of the soft gluons and transport of their energy outside of the jet cone. Clearly, the effect will be most pronounced for large radii $(R=0.7)$ that contain a significant fraction of the medium-induced parton shower. For small radii $(R=0.2)$ the effect is negligible. Dissipation of the parton shower energy, of course, still occurs. However, owing to the broad distribution of the medium-induced shower, which is now verified to $\mathcal{O}\left(\left(\alpha_{s}^{\text {med }}\right)^{2}\right)$ [35], this effect is negligible. There 
simply isn't much of the medium-induced shower left inside the jet cone.

Next, we turn to the dependence of the nuclear suppression of jet production on the coupling between the jet and the strongly-interacting medium. We note that theoretical predictions for inclusive jet suppression, modification of the di-jet asymmetry distributions, and $Z^{0}$ tagged and $\gamma$-tagged jet momentum imbalance distributions with coupling constant in the range $g^{\text {med }}=2.0-2.2$ have compared favourably with the experimental findings [8, 9]. We also perform the calculation for an average number of participants $N_{\text {part }}=120$ and include isospin effects and cold nuclear matter energy loss [37]. Note, that at $p_{T}$ up to $\sim 20 \mathrm{GeV}$ in $\sqrt{s_{N N}}=5.08 \mathrm{TeV}$ $\mathrm{p}+\mathrm{Pb}$ collisions at the LHC CNM effects appear to be negligible [39]. Above $20 \mathrm{GeV}$ currently there are no experimental measurements in $\mathrm{p}+\mathrm{A}$ reactions at the LHC. The effect of CNM energy loss is less than $~ 15 \%$ suppression of b-jet production in minimum bias $\mathrm{Pb}+\mathrm{Pb}$ collisions (corresponding roughly to a $7.5 \%$ effect in minimum bias $\mathrm{p}+\mathrm{A}$ collisions). It also leads to a weaker $p_{T}$ dependence of $R_{A A}$. Our simulations are done in the pseudorapidity interval $|\eta|<2$. We used three different couplings between the jet and the medium: $g^{\text {med }}=1.8$ (blue dot-dot-dashed line), $g^{\text {med }}=2.0$ (green solid line), $g^{\text {med }}=2.2$ (red dashed line). The dependence on $g^{\text {med }}$ can clearly be identified if experimental accuracy comparable to that of light jet $R_{A A}$ measurements can be achieved. Shown in Fig. 5 is a very preliminary CMS measurement of b-jet $R_{A A}$ for $p_{T}>100 \mathrm{GeV}$ in the pseudorapidity region $|\eta|<2$. This preliminary result is for minimum bias collisions. We observe that there is agreement between the simulations and the CMS data point within the large error bars for $g^{\text {med }}$. It is not surprising

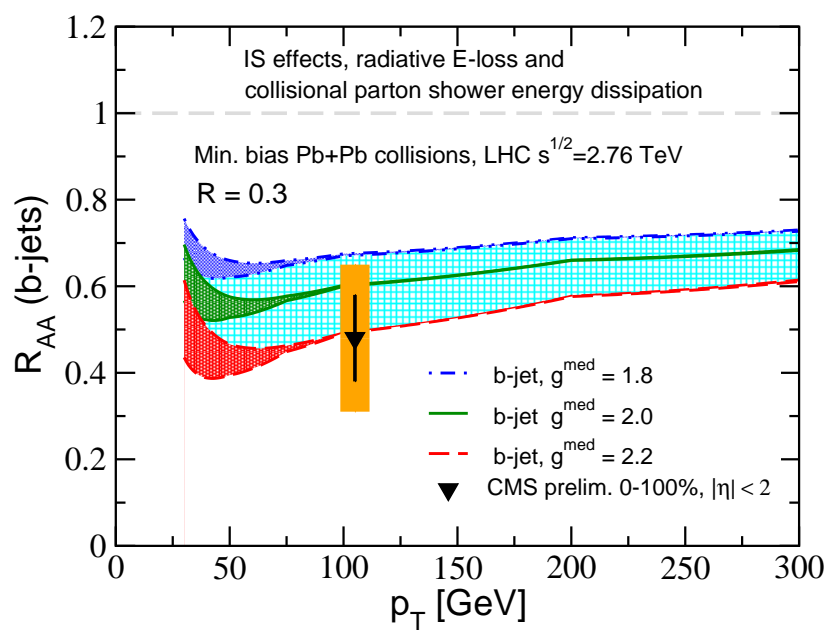

FIG. 5. The $p_{T}$-dependent suppression of the b-jet production cross section in minimum-bias $\sqrt{s_{N N}}=2.76 \mathrm{TeV} \mathrm{Pb}+\mathrm{Pb}$ collisions at the LHC is shown for 3 different couplings between the jet and the medium, $g^{\text {med }}=1.8,2.0,2.2$. The pseudeorapidity interval is $|\eta|<2$ and the jet radius is $R=0.3$. Preliminary data is from the CMS collaboration.

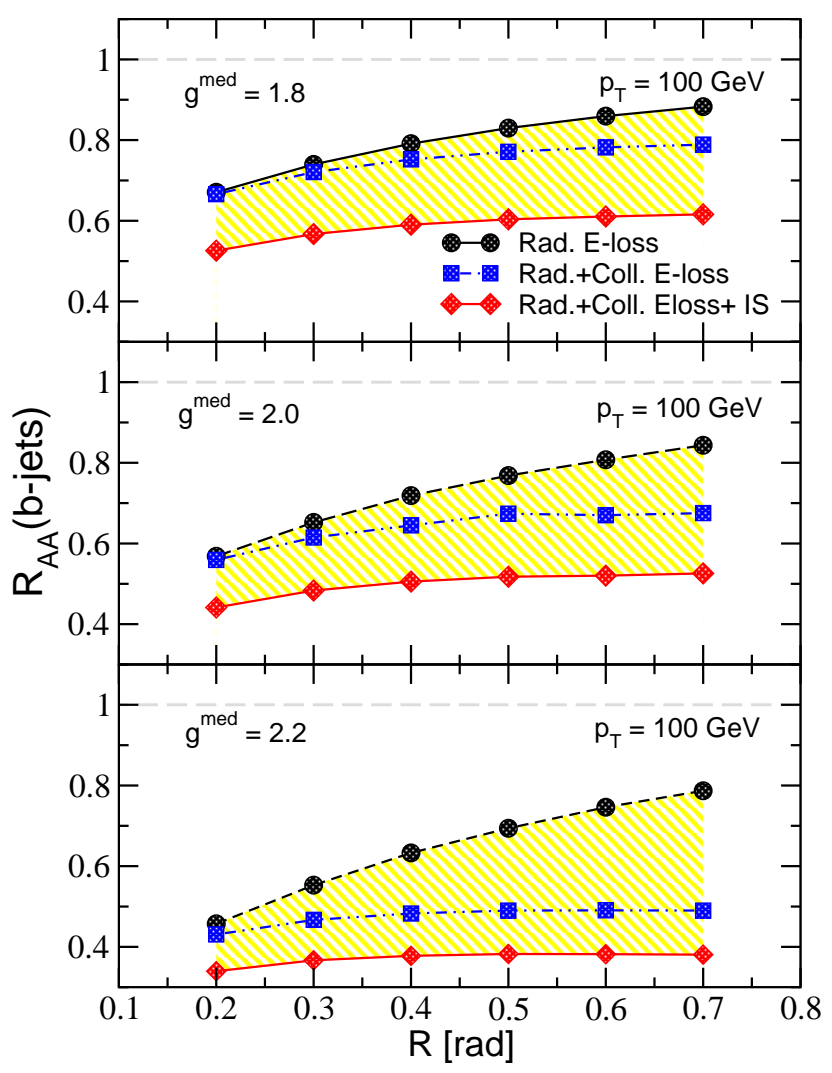

FIG. 6. $R$ dependence of $p_{T}=100 \mathrm{GeV}$ b-jet suppression as a function of initial-state and final-state effects. Upper panel is for a coupling between the jet and the medium $g^{\text {med }}=1.8$, middle panel is for $g^{\text {med }}=2$, and lower panel is for $g^{\text {med }}=2.2$.

that the suppression of b-jets is similar to the suppression of light quark jets of the same transverse momentum. At $p_{T} \gtrsim 75 \mathrm{GeV}$ the effect of the heavy quark mass, $m_{b}=4.5 \mathrm{GeV}$ to $2 m_{b}=9 \mathrm{GeV}$, disappears in the coherent energy loss regime. On the other hand, the fact that $g \rightarrow b \bar{b}$ system can retain the quantum numbers of a gluon implies energy loss considerably larger than that of a quark. What would be surprising is if the suppression of b-jet in minimum-bias reaction will be equal or larger than the suppression of the light jets in central $\mathrm{Pb}+\mathrm{Pb}$ reactions.

Last but not least, we study the radius dependence and the influence of cold and hot nuclear matter effects on the b-jet production in central $\mathrm{Pb}+\mathrm{Pb}$ collisions. Our results are shown in Fig. 6 for $g^{\text {med }}=1.8$ (upper panel), $g^{\text {med }}=2.0$ (middle panel), and $g^{\text {med }}=2.2$ (lower panel). For definitiveness we fix the jet $p_{T}=100 \mathrm{GeV}$. The slope of the $R_{A A}(R)$ can help constrain the relative contribution of radiative and collisional energy loss processes (redistribution of the hard scattered parton energy between a vacuum and a medium-induced parton shower and collisional dissipation of the medium-induced parton shower energy in the QGP). CNM effects do not change this slope but rather lead to a constant $\sim 15-20 \%$ suppression. This creates a degeneracy between the strength of the 
coupling between the jet and the medium and CNM effects. For example, the simulated $R_{A A}$ with $g^{\text {med }}=2.2$ in the absence of initial state energy loss is practically the same as the suppression with $g^{\text {med }}=2.0$ that includes these inelastic effects. This degeneracy cannot be resolved based on $\mathrm{Pb}+\mathrm{Pb}$ measurements alone. b-jet (or even light jet) experimental analysis in $\mathrm{p}+\mathrm{Pb}$ reactions is needed to constrain CNM effects to high transverse momentum at the LHC.

\section{CONCLUSIONS}

In summary, we presented theoretical predictions for the nuclear suppression of the inclusive b-jet differential cross section in $\mathrm{Pb}+\mathrm{Pb}$ collisions at $\sqrt{s_{N N}}=2.76 \mathrm{TeV}$ at the LHC. Our results combine b-jet production in $\mathrm{p}+\mathrm{p}$, simulated using Pythia 8, with the cold nuclear matter and QGP effects. The nuclear modification factor $R_{A A}$, as a function of the jet transverse momentum $p_{T}$ and the jet radius parameter $R$, can be compared to the upcom- ing LHC experimental data. We find that, for $p_{T} \gtrsim 75$ $\mathrm{GeV}$, the nuclear-induced attenuation of b-jets is comparable to the one observed for light jets. The disappearance of the heavy quark mass effects at high transverse momentum is a direct consequence of the fully-coherent final state energy loss in the QGP. The detailed radius dependence of $R_{A A}$ at fixed $p_{T}$ provides information for the relative significance of the medium-induced parton shower formation and the dissipation of its energy in the strongly-interacting plasma. Experimental measurements of b-jet production in heavy ion collisions will, thus, provide unique new insights into heavy flavor dynamics in dense QCD matter.

\section{ACKNOWLEDGMENTS}

We thank Kun Liu, Tao Liu, Yaxian Mao, Feng Wei, and Zhenyu Ye for very helpful discussions. This research is supported by the US Department of Energy, Office of Science, and in part by the LDRD program at LANL.
[1] J. M. Campbell, J. W. Huston and W. J. Stirling, Rept. Prog. Phys. 70, 89 (2007); S. D. Ellis, J. Huston, K. Hatakeyama, P. Loch and M. Tonnesmann, Prog. Part. Nucl. Phys. 60, 484 (2008).

[2] G. Aad et al. [ATLAS Collaboration], Eur. Phys. J. C 71, 1512 (2011); Phys. Rev. D 86, 014022 (2012).

[3] S. Chatrchyan et al. [CMS Collaboration], Phys. Lett. B 700, 187 (2011); Phys. Rev. Lett. 107, 132001 (2011); arXiv:1212.6660 [hep-ex].

[4] B. Abelev et al. [ALICE Collaboration], arXiv:1301.3475 [nucl-ex].

[5] I. Vitev, S. Wicks and B. -W. Zhang, JHEP 0811, 093 (2008); I. Vitev and B. -W. Zhang, Phys. Rev. Lett. 104, 132001 (2010).

[6] G. Aad et al. [Atlas Collaboration], Phys. Rev. Lett. 105, 252303 (2010); Phys. Lett. B 719, 220 (2013).

[7] S. Chatrchyan et al. [CMS Collaboration], Phys. Rev. C 84, 024906 (2011); Phys. Lett. B 712, 176 (2012).

[8] R. B. Neufeld, I. Vitev and B. -W. Zhang, Phys. Rev. C 83, 034902 (2011); R. B. Neufeld and I. Vitev, Phys. Rev. Lett. 108, 242001 (2012).

[9] Y. He, I. Vitev and B. -W. Zhang, Phys. Lett. B 713, 224 (2012), W. Dai, I. Vitev and B. -W. Zhang, Phys. Rev. Lett. 110, 142001 (2013).

[10] G. -Y. Qin and B. Muller, Phys. Rev. Lett. 106, 162302 (2011) [Erratum-ibid. 108, 189904 (2012)]. G. -Y. Qin, arXiv:1210.6610 [hep-ph].

[11] X. -N. Wang and Y. Zhu, arXiv:1302.5874 [hep-ph]; G. L. Ma, arXiv:1304.2841 [nucl-th].

[12] C. Young, B. Schenke, S. Jeon and C. Gale, Phys. Rev. C 84, 024907 (2011); I. P. Lokhtin, A. V. Belyaev and A. M. Snigirev, Eur. Phys. J. C 71, 1650 (2011).

[13] S. S. Adler et al. [PHENIX Collaboration], Phys. Rev. Lett. 96, 032301 (2006); A. Adare et al. [PHENIX Collaboration], Phys. Rev. Lett. 98, 172301 (2007).
[14] B. Abelev et al. [ALICE Collaboration], JHEP 1209, 112 (2012); Phys. Rev. Lett. 109, 112301 (2012).

[15] A. Adil and I. Vitev, Phys. Lett. B 649, 139 (2007) hep-ph/0611109]; R. Sharma, I. Vitev and B. -W. Zhang, Phys. Rev. C 80, 054902 (2009).

[16] S. Wicks, W. Horowitz, M. Djordjevic and M. Gyulassy, Nucl. Phys. A 784, 426 (2007).

[17] H. van Hees, M. Mannarelli, V. Greco and R. Rapp, Phys. Rev. Lett. 100, 192301 (2008).

[18] G. -Y. Qin and A. Majumder, Phys. Rev. Lett. 105, 262301 (2010).

[19] P. B. Gossiaux and J. Aichelin, Phys. Rev. C 78, 014904 (2008).

[20] Z. -B. Kang and I. Vitev, Phys. Rev. D 84, 014034 (2011); T. Stavreva, F. Arleo and I. Schienbein, JHEP 1302, 072 (2013).

[21] T. Sjostrand, S. Mrenna and P. Z. Skands, Comput. Phys. Commun. 178, 852 (2008).

[22] J. Pumplin, D. R. Stump, J. Huston, H. L. Lai, P. M. Nadolsky and W. K. Tung, JHEP 0207, 012 (2002).

[23] M. Cacciari, G. P. Salam and G. Soyez, JHEP 0804, 063 (2008).

[24] M. Cacciari, G. P. Salam and G. Soyez, Eur. Phys. J. C 72, 1896 (2012).

[25] S. Chatrchyan et al. [CMS Collaboration], JHEP 1204, 084 (2012) arXiv:1202.4617 [hep-ex]].

[26] E. Norrbin and T. Sjostrand, Eur. Phys. J. C 17, 137 (2000) hep-ph/0005110.

[27] It has been shown recently [28] that the results between the fixed flavor scheme and variable flavor scheme are consistent for a large range of kinematics at LHC.

[28] F. Maltoni, G. Ridolfi and M. Ubiali, JHEP 1207, 022 (2012) [Erratum-ibid. 1304, 095 (2013)] arXiv:1203.6393 [hep-ph]]. 
[29] Y. L. Dokshitzer and D. E. Kharzeev, Phys. Lett. B 519, 199 (2001); B. W. Zhang, E. Wang and X. N. Wang, Phys. Rev. Lett. 93, 072301 (2004); M. Djordjevic and M. Gyulassy, Nucl. Phys. A 733, 265 (2004).

[30] R. Sharma and I. Vitev, Phys. Rev. C 87, 044905 (2013).

[31] A. Banfi, G. P. Salam and G. Zanderighi, JHEP 0707, 026 (2007) arXiv:0704.2999 [hep-ph]]; G. Aad et al. [ATLAS Collaboration], Eur. Phys. J. C 73, 2301 (2013) arXiv:1210.0441 [hep-ex]].

[32] T. Aaltonen et al. [CDF Collaboration], Phys. Rev. D 78, 072005 (2008) arXiv:0806.1699 [hep-ex]].

[33] G. Ovanesyan and I. Vitev, JHEP 1106, 080 (2011).

[34] I. Vitev, Phys. Lett. B 630, 78 (2005); G. Ovanesyan and I. Vitev, Phys. Lett. B 706, 371 (2012).
[35] M. Fickinger, G. Ovanesyan and I. Vitev, arXiv:1304.3497 [hep-ph].

[36] R. B. Neufeld and I. Vitev, Phys. Rev. C 86, 024905 (2012); R. B. Neufeld, Phys. Rev. D 83, 065012 (2011).

[37] I. Vitev, Phys. Rev. C 75, 064906 (2007); R. B. Neufeld, I. Vitev and B. -W. Zhang, Phys. Lett. B 704, 590 (2011).

[38] I. Vitev, J. T. Goldman, M. B. Johnson and J. W. Qiu, Phys. Rev. D 74, 054010 (2006).

[39] B. Abelev et al. [ALICE Collaboration], Phys. Rev. Lett. 110, 082302 (2013); Z. -B. Kang, I. Vitev and H. Xing, Phys. Lett. B 718, 482 (2012); J. L. Albacete, N. Armesto, R. Baier, G. G. Barnafoldi, J. Barrette, S. De, W. -T. Deng and A. Dumitru et al., Int. J. Mod. Phys. E Vol. 22, 1330007 (2013). 

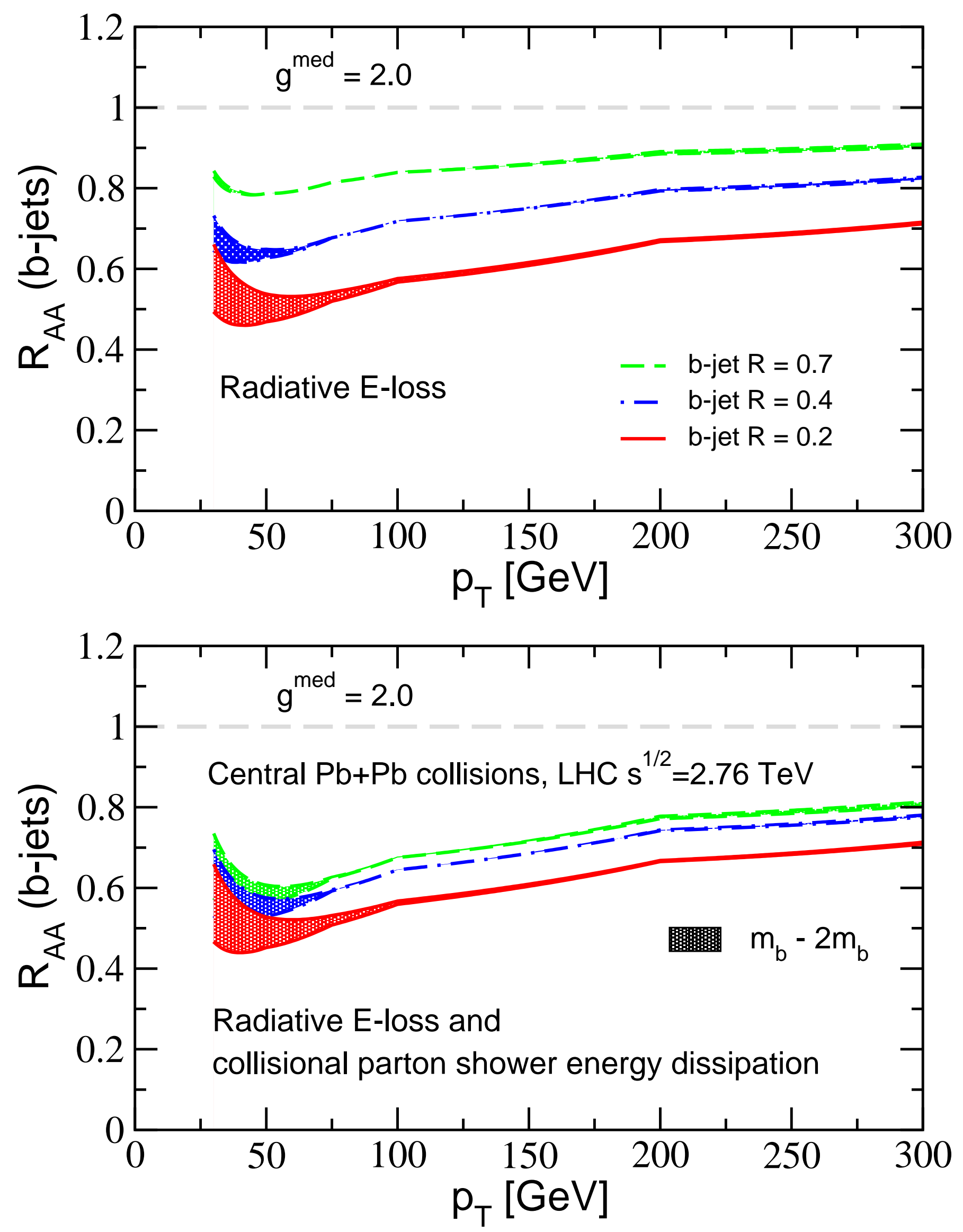